\title{
An investigational RNAi therapeutic targeting Factor XII (ALN-F12) for the treatment of hereditary angioedema
}

\author{
JINGXUAN LIU, JUNE QIN, ANNA BORODOVSKY, TIMOTHY RACIE, ADAM CASTORENO, MARK SCHLEGEL, \\ MARTIN A. MAIER, TRACY ZIMMERMAN, KEVIN FITZGERALD, JAMES BUTLER, and AKIN AKINC
}

Alnylam Pharmaceuticals, Cambridge, Massachusetts 02142, USA

\begin{abstract}
Hereditary angioedema (HAE) is a genetic disorder mostly caused by mutations in the C1 esterase inhibitor gene (C1INH) that results in poor control of contact pathway activation and excess bradykinin generation. Bradykinin increases vascular permeability and is ultimately responsible for the episodes of swelling characteristic of HAE. We hypothesized that the use of RNA interference (RNAi) to reduce plasma Factor XII (FXII), which initiates the contact pathway signaling cascade, would reduce contact pathway activation and prevent excessive bradykinin generation. A subcutaneously administered GalNAcconjugated small-interfering RNA (siRNA) targeting F12 mRNA (ALN-F12) was developed, and potency was evaluated in mice, rats, and cynomolgus monkeys. The effect of FXII reduction by ALN-F12 administration was evaluated in two different vascular leakage mouse models. An ex vivo assay was developed to evaluate the correlation between human plasma FXII levels and high-molecular weight kininogen (HK) cleavage. A single subcutaneous dose of ALN-F12 led to potent, dose-dependent reduction of plasma FXII in mice, rats, and NHP. In cynomolgus monkeys, a single subcutaneous dose of ALN-F12 at $3 \mathrm{mg} / \mathrm{kg}$ resulted in $>85 \%$ reduction of plasma FXII. Administration of ALN-F12 resulted in dose-dependent reduction of vascular permeability in two different mouse models of bradykinin-driven vascular leakage, demonstrating that RNAi-mediated reduction of FXII can potentially mitigate excess bradykinin stimulation. Lastly, ex vivo human plasma HK cleavage assay indicated FXII-dependent bradykinin generation. Together, these data suggest that RNAi-mediated knockdown of FXII by ALN-F12 is a potentially promising approach for the prophylactic treatment of HAE.
\end{abstract}

Keywords: hereditary angioedema; GaINAc-siRNA; Factor XII; bradykinin; vascular permeability; HK cleavage

\section{INTRODUCTION}

Hereditary angioedema (HAE) is an autosomal dominant disease characterized by recurrent episodes of potentially life-threatening angioedema, with particular focus on the skin, gastrointestinal submucosa, and upper airway (Zuraw 2010; Zuraw and Christiansen 2011; Walford and Zuraw 2014). The prevalence of HAE ranges from $1: 10,000$ to $1: 50,000$ individuals. HAE is primarily caused by deficiency or malfunction of the $\mathrm{C} 1$ esterase inhibitor (C1INH) (type I and II, respectively) (Bork and Davis-Lorton 2013). C1INH regulates the activation of complement and intrinsic coagulation (contact activation pathway) by inhibiting a number of plasma proteases including C1r, C1s, plasma

Corresponding author: jingxuanliu@alnylam.com

Abbreviations: GalNAc-siRNA, GalNAc-conjugated short-interfering RNA; ALN-F12, GalNAc-siRNA targeting liver specific Factor XII; $\mathrm{HAE}$, Hereditary angioedema; $\mathrm{C} 1 \mathrm{INH}, \mathrm{C} 1$ esterase inhibitor; $\mathrm{ACEi}$, angiotensin-converting enzyme inhibitor; F12, F12 gene; FXII, Factor XII protein; FXIla, Activated Factor XII protein; PK, Plasma prekallikrein; PKa, Plasma kallikrein; HK, high-molecular weight kininogen; SC, subcutaneous; IV, intravenous

Article is online at http://www.rnajournal.org/cgi/doi/10.1261/rna. 068916.118. kallikrein, FXIla and FXla. With respect to HAE, plasma kallikrein and FXIla are of particular interest as they are key regulators in the kallikrein-kinin branch of the contact pathway that regulates bradykinin production. In HAE patients with $\mathrm{C} 1 \mathrm{INH}$ deficiency, the absence of a functional "brake" leads to uncontrolled kallikrein-kinin pathway activation and the generation of excessive amounts of bradykinin, a key mediator for vascular permeability and subsequent angioedema (Carugati et al. 2001; Nussberger et al. 2002; Pappalardo et al. 2002).

FXII, also known as Hageman factor (OMIM 234000), is a protein zymogen primarily produced in the liver that sits atop the kallikrein-kinin pathway (Schmaier 2000). When it comes into contact with negatively GalNAc-siRNA charged surfaces, FXII auto-activates into active serine protease FXIla. Although the precise trigger for FXII activation in patients with HAE is currently unknown, various in vivo

(C) 2019 Liu et al. This article is distributed exclusively by the RNA Society for the first 12 months after the full-issue publication date (see http://rnajournal.cshlp.org/site/misc/terms.xhtml). After 12 months, it is available under a Creative Commons License (Attribution-NonCommercial 4.0 International), as described at http:// creativecommons.org/licenses/by-nc/4.0/. 
activators of FXII have been identified, including heparin, misfolded protein aggregates, polyphosphates and nucleic acids (Müller et al. 2009; Björkqvist et al. 2014). Upon activation, the direct downstream targets of FXIla include Factor XI (FXI) and plasma prekallikrein (PK). The FXIla cleavage of FXI into FXla drives the activation of the intrinsic coagulation pathway and gives rises to fibrin formation. Activation of PK by FXIla results in the production of plasma kallikrein serine protease ( $\mathrm{PKa}$ ) which then cleaves HK. Cleavage of $\mathrm{HK}$ leads to the release of bradykinin, a nine amino acid signaling peptide that controls vascular permeability through its interaction with bradykinin-2 receptor (B2R); the remaining polypeptide, two-chain HK, is comprised of two polypeptide chains (heavy and light) linked by a disulfide bond (Zhang et al. 2000). In patients, it is the uncontrolled generation of excess bradykinin that leads to HAE attacks.

As an initiator of the bradykinin generation pathway, FXII appears to be an intriguing target for HAE treatment. In line with this therapeutic concept, the $\mathrm{F} 12-46 \mathrm{C} / \mathrm{T}$ polymorphism (rs1801020) results in lower plasma FXII levels and is associated with a delay in HAE disease onset and decreased need for long-term prophylaxis (Kanaji et al. 1998; Speletas et al. 2015). In addition to the two classical types of HAE with diminished C1INH function (type I and II), some HAE patients present normal C1INH functionality levels (type III HAE). Among this population, mutations in the $F 12$ gene have been reported to lead to loss of glycosylation, which led to increased contact-mediated autoactivation of zymogen FXII, resulting in excessive activation of the kallikrein-kinin pathway (Cichon et al. 2006; Bork 2013; Björkqvist et al. 2015; de Maat et al. 2016). In the European type III HAE population, F12 mutations were identified in up to $25 \%$ of cohorts (Bork et al. 2009). Both aspects of the genetic evidence support the therapeutic concept of targeting F12 for HAE treatment.

To evaluate the effect of knocking down F12 in the alleviation of HAE, we developed ALN-F12, an F12 targeting small-interfering RNA (siRNA) conjugated with trivalent $\mathrm{N}$-acetylgalactosamine (GalNAc) ligand (Nair et al. 2014; Matsuda et al. 2015) to allow hepatocyte-specific delivery after subcutaneously dosing (Fig. 1A). Currently, the GalNAc-siRNA technology has been used in multiple ongoing clinical programs (Fitzgerald et al. 2017a,b; Zimmermann et al. 2017). We hypothesized that reduction of plasma FXII levels by ALNF12 can compensate for the defect resulting from the loss of functional $\mathrm{C} 1 \mathrm{INH}$ and alleviate excess bradykinin generation (Fig. 1B).

\section{RESULTS}

\section{In vitro and in vivo screenings for ALN-F12}

A set of 79 siRNAs targeting FXII bearing GalNAc-ligand were tested for in vitro activity by transfection in the human hepatoma Hep3B cell line (Supplemental Table S1A). From this set, 12 efficacious siRNAs were selected for dose-response assessment (Supplemental Table S1B). Following the identification of three potent lead sequences, two chemically modified analogs targeting the same transcript site were prepared for each lead and all six siRNAs were evaluated for in vivo activity in mice (Supplemental Table $\mathrm{S} 1 \mathrm{C})$. ALN-F12 was selected based on both in vitro and in vivo screenings.

To assess the potential for off-target effects, a bioinformatic search against the human transcriptome was conducted for ALN-F12. The top 31 predicted potential offtarget transcripts were evaluated in Hep3B cells, and none of the predicted potential off-target transcripts were inhibited substantially by ALN-F12 (data not shown).

\section{ALN-F12 demonstrates robust potency and durability in rodents}

To evaluate the potency and stability in vivo, GalNAc conjugated siRNA duplex ALN-F12 was subcutaneously dosed into female $\mathrm{C} 57 \mathrm{BL} / 6$ mice at 0.3 or $1.0 \mathrm{mg} / \mathrm{kg}$.
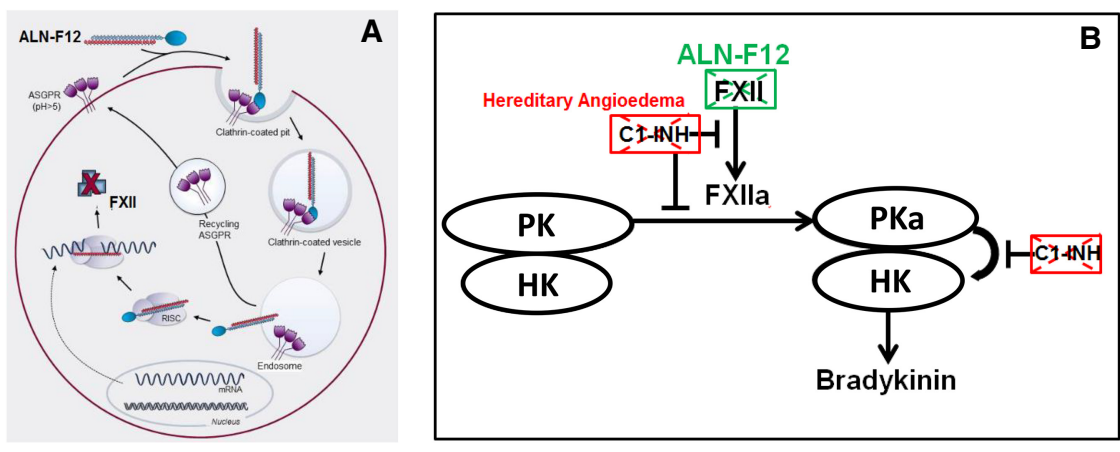

FIGURE 1. ALN-F12 for HAE prophylaxis: therapeutic hypothesis. (A) ALN-F12 silences hepatic F12 gene expression. N-acetylgalactosamine (GalNAc) conjugated siRNA duplex ALN-F12 specifically binds hepatocytes via the asialoglycoprotein receptor (ASGPR). Following calthrinmediated endocytosis and endosomal escape, ALN-F12 is unwound and loaded into RISC (RNA-induced silencing complex) and specifically recognizes and degrades F12 mRNA, leading to a reduction of secreted Factor XII (FXII) protein levels in plasma. (B) Conversion of FXII to FXIla initiates an enzymatic signaling cascade that results in the production of bradykinin, a peptide signal that controls vascular permeability. $\mathrm{C} 1$ esterase inhibitor (C1INH), which binds and inhibits FXIla and plasma kallikrein (PKa), negatively regulates this process. In HAE patients, loss of $\mathrm{C} 1 \mathrm{INH}$ activity leads to excess bradykinin generation, resulting in increased vascular permeability and subsequent edema. RNAi-mediated knockdown of F12 gene expression by ALN-F12 would decrease circulating FXII protein levels and therefore preventing excess bradykinin generation. 
The plasma total FXII levels were measured by ELISA and normalized to the average of the PBS control group. A single dose of ALN-F12 in mice led to dose-dependent, maximum, suppression of circulating FXII of $~ 51 \%$ at $0.3 \mathrm{mg} /$ $\mathrm{kg}$ and $93 \%$ at $1 \mathrm{mg} / \mathrm{kg}$ on Day 10 . FXII levels returned to baseline by $\sim 64 \mathrm{~d}$ after treatment (Fig. 2A). Similarly, a single subcutaneously dose of ALN-F12 in female Sprague-Dawley rats led to robust, dose-dependent reduction of plasma FXII (Fig. 2B). The suppression of liver F12 mRNA levels is comparable with the reduction of plasma FXII protein levels in both mice and rats (Supplemental Fig. S1).

\section{RNAi-mediated FXII reduction alleviated ACE inhibitor-induced vascular leakage}

It has been demonstrated previously that a vasodilator angiotensin-converting enzyme (ACE) inhibitor, such as captopril, can increase vascular permeability by blocking the breakdown of bradykinin (Migdalof et al. 1984; Han et al. 2002). To examine the effect of FXII suppression on captopril-induced vascular leakage, ALN-F12 was dosed in
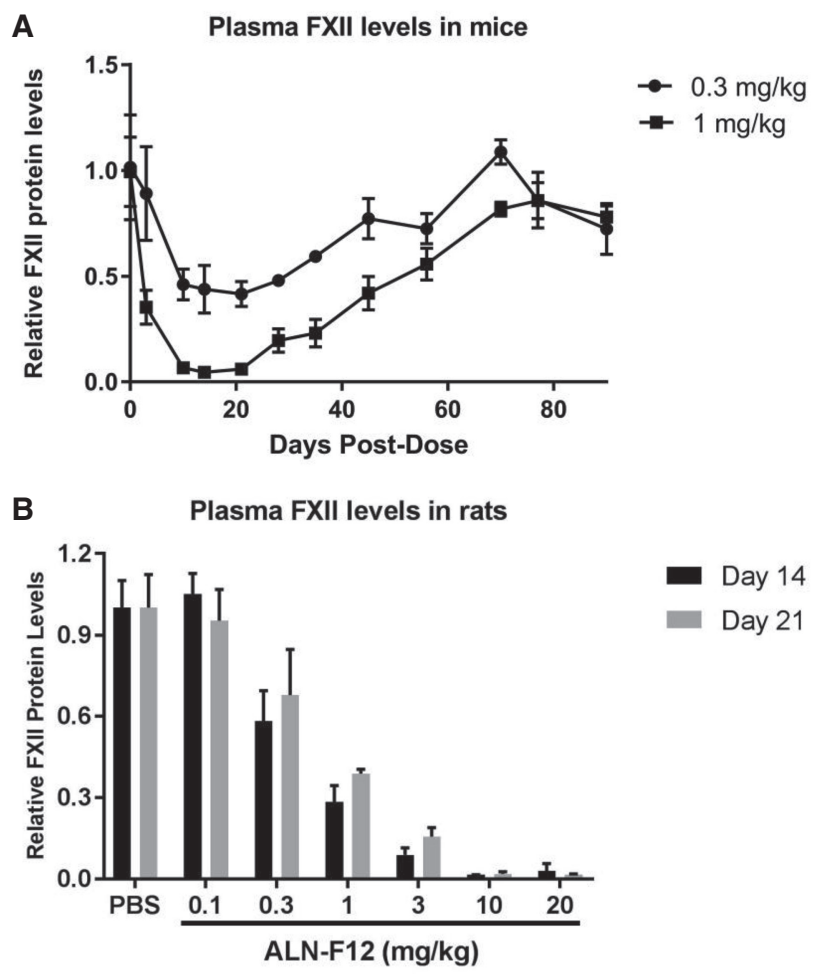

FIGURE 2. ALN-F12 dose-dependent knockdown of circulating FXII in mice and rats. (A) Female C57BL/6J mice were subcutaneously administered a single dose of ALN-F12 ( $n=3$ per group). Plasma FXII levels were measured by ELISA and normalized to PBS control group. (B) Female Sprague-Dawley rats were subcutaneously administered a single dose of ALN-F12 ( $n=3$ per group). Plasma FXII levels were measured by ELISA and normalized to the average of PBS control group. Error bars are SD. female C57BL/6 mice at different levels $(0.1,0.3,1$, and $3 \mathrm{mg} / \mathrm{kg}$ ) $7 \mathrm{~d}$ before captopril induction to allow maximum knockdown. Vascular flow was traced by IV injection of Evans blue dye, a high affinity inert binder to plasma albumin. IV injection of $2.5 \mathrm{mg} / \mathrm{kg}$ of captopril led to dramatic vascular leakage within $30 \mathrm{~min}$ represented by decreased Evans blue dye extraction from the circulation system and increased extraction from peripheral tissue (Fig. 3A,B). Suppression of plasma FXII mitigated the captoprilinduced vascular leakage in a dose-dependent manner, represented by increased Evans blue dye extraction from the circulation and decreased from the intestine. Interestingly, in this model, 50\% F12 mRNA reduction completely prevented vascular leakage induced by captopril (Fig. 3C,D). The same studies were also performed by knocking down contact activation pathway downstream components, PK or HK to different levels using GalNAcsiRNAs specifically targeting each of them. In contrast to F12 mRNA, 75\% silencing of PK mRNA and $>90 \%$ silencing of HK mRNA was necessary to prevent captopril-induced vascular permeability (Fig. 3E,F; Supplemental Figs. S2, S3). No synergistic effect was observed by knocking down both PK and FXII (data not shown).

\section{FXII knockdown mitigated mustard oil-induced vascular leakage in C1INH knockdown mice}

Clinically, both type I and type II HAE result from $\mathrm{C} 1 \mathrm{INH}$ deficiency or dysfunction. Although C1INH-deficient mice do not develop symptomatic angioedema, they have increased baseline vascular permeability and higher sensitivity to the topical application of inflammatory stimulator mustard oil (Han et al. 2002). To generate C1INH knockdown mice, we developed a GalNAc-siRNA duplex targeting mouse $\mathrm{C} 1 \mathrm{INH}$ (C1INH-siRNA). C1INH is mainly synthesized in the liver, thereby allowing efficacious suppression of plasma $\mathrm{C} 1 \mathrm{INH}$ by GalNAc-conjugated C1INH-siRNA. A single SC dose of C1INH-siRNA at 10 $\mathrm{mg} / \mathrm{kg}$ led to $>95 \%$ decrease of liver C1INH mRNA levels (Supplemental Fig. S4B). Inflammatory stimulator, mustard oil, was topically applied to the right ear to increase vascular leakage. The left ear of each animal served as a control. Consistent with the homozygous $\mathrm{C} 1 \mathrm{INH}$-deficient mice, $10 \mathrm{mg} / \mathrm{kg} \mathrm{C} 1 \mathrm{INH}$-siRNA-treated mice also displayed higher baseline vascular permeability and increased sensitivity to mustard oil stimulation (Fig. 4A). Knocking down of FXII by ALN-F12 in C1INH knockdown mice mitigated the vascular fluid leakage in a dose-dependent manner, as demonstrated by decreased Evans blue dye extraction of both untreated and mustard oil stimulated ears (Fig. 4A,B). Knocking down other components in the contact activation pathway, such as $\mathrm{PK}$ and $\mathrm{HK}$, also mitigated the vascular leakage in this model (Supplemental Fig. S4). It is worth noting that in this model, in contrast to the ACEi-induced 

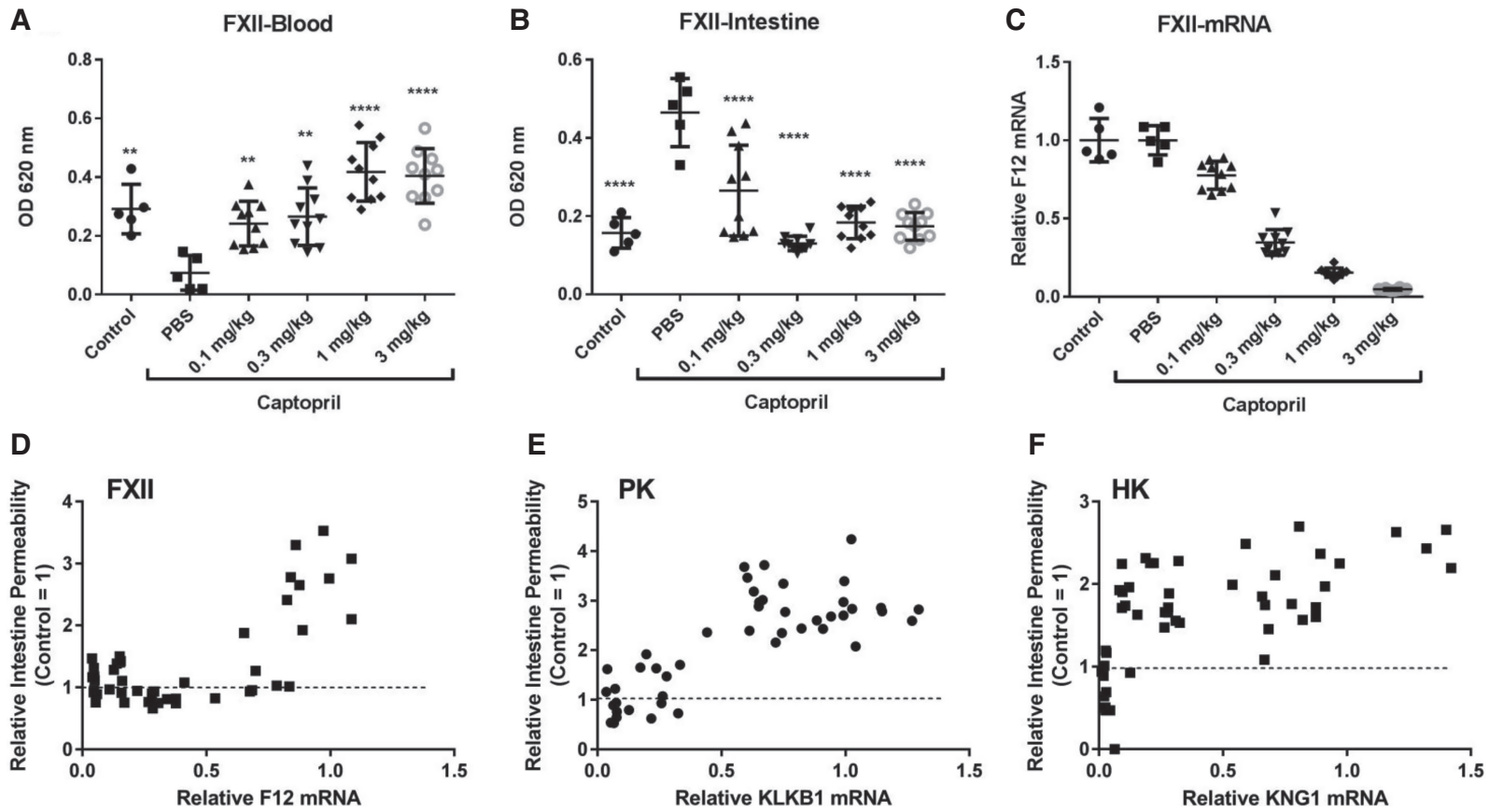

FIGURE 3. Inhibition of ACE inhibitor-induced vascular permeability following a single dose subcutaneous administration of ALN-F12. Female C57BL/6J mice were subcutaneously administered a single dose of ALN-F12 at dose levels of 0, 0.1, 0.3, 1, or $3 \mathrm{mg} / \mathrm{kg}$ ( $n=10 \mathrm{per}$ group). Seven days after administration, animals were IV injected with the ACE inhibitor captopril $(2.5 \mathrm{mg} / \mathrm{kg})$; the vascular permeability tracer, Evans blue dye $(30 \mathrm{mg} / \mathrm{kg}$ ), was IV injected $15 \mathrm{~min}$ after captopril injection. Animals were euthanized $15 \mathrm{~min}$ after Evans blue dye injection. Blood ( $A$ ) and intestine (B) were collected to evaluate Evans blue extravasation; livers were collected for $F 12$ mRNA analysis $(C)$. In panels $A, B$, and $C$, symbols denote data from individual animals, the group mean \pm SD are also plotted. Statistical analysis performed using one-way ANOVA with Dunnett's post-hoc test with respect to PBS-treated animals in the captopril treatment group. $\left(^{* *}\right) P<0.01 ;\left(^{* * * *}\right) P<0.0001$. A correlation of intestinal Evans blue uptake and F12 mRNA levels (D). Panels $E$ and $F$ are the correlation of intestinal Evans blue extraction and PK (and HK) mRNA levels (Supplemental Figs. S2, S3).

vascular leakage model, suppression of FXII, PK, and HK all yielded a similar outcome.

\section{ALN-F12 led to dose-dependent, sustainable suppression of plasma FXII in nonhuman primate (NHP)}

ALN-F12 is also designed to target $\mathrm{NHP}$ and human $\mathrm{F} 12$ genes. To determine the efficacy and durability of ALN-F12 in NHP, a single dose of ALN-F12 was subcutaneously administered at $0.1,0.3,1$, or $3 \mathrm{mg} / \mathrm{kg}$ in female cynomolgus monkeys ( $n=3$ per group). Plasma was collected at predose (Days 5, 3, and 1) and a series of time points after dosing. Levels of circulating FXII protein were measured by plasma ELISA and normalized to the pre-dose average of each individual animal. A single SC dose
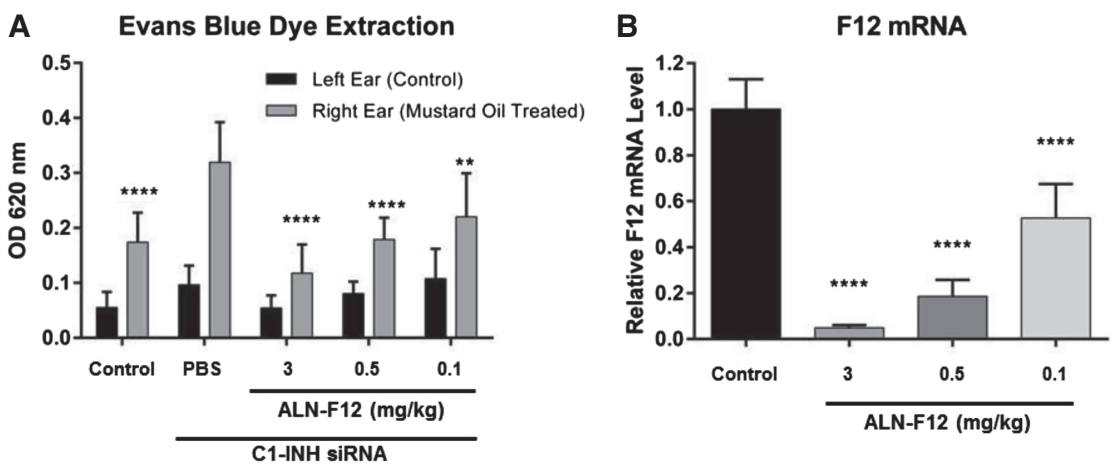

FIGURE 4. ALN-F12-rescued mustard oil-induced vascular permeability in a C1INH knockdown mouse model. Female CD1 mice were subcutaneously dosed with a siRNA targeting $\mathrm{C} 1 \mathrm{INH}(10 \mathrm{mg} / \mathrm{kg})$ and $\mathrm{ALN}-\mathrm{F} 12$ at different dose levels ( $n=10$ per group). Seven days post-administration, Evans blue dye (30 mg/kg) was given IV, and $5 \%$ mustard oil was topically applied to the right ear, followed $15 \mathrm{~min}$ later by a second application. The animals were euthanized 15 min after the second application of mustard oil. Both right (mustard oil-treated) and left (control) ears were collected for dye extraction (A). Livers were collected for mRNA measurements (B). Group mean \pm SD are plotted. Statistical analysis in Panel A performed using two-way ANOVA with Tukey's post-hoc test; asterisks indicate statistical comparisons to mustard oil-treated ears in the PBS control group. Statistical analysis in Panel B performed using one-way ANOVA with Dunnett's post-hoc test; statistical comparisons to PBS-treated animals. For both analyses: $\left(^{* *}\right) P<0.01 ;(* * * *) P<0.0001$. 

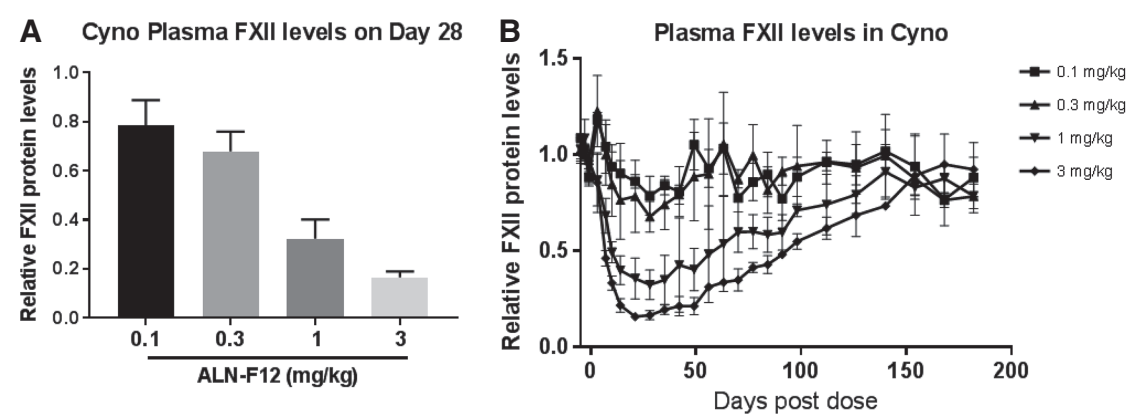

FIGURE 5. ALN-F12 dose-dependent reduction of circulating FXII in female cynomolgus monkeys. Female cynomolgus monkeys were subcutaneously administered a single dose of ALN-F12 ( $n=3$ per group). Plasma FXII levels were measured by ELISA and normalized to the average of individual animal's pre-dose average of Days $-5,-3$, and -1 (error bars = SD). Panel A represents the relative plasma FXII level on Day 28 post-dosing; Panel B depicts the relative FXII levels at all collected time points for all dosing groups.

of ALN-F12 led to dose-dependent suppression of circulating FXII in cynomolgus monkeys with maximal suppression of $\sim 21 \%$ at $0.1 \mathrm{mg} / \mathrm{kg}, 32 \%$ at $0.3 \mathrm{mg} / \mathrm{kg}, 68 \%$ at 1 $\mathrm{mg} / \mathrm{kg}$, and $83 \%$ at $3 \mathrm{mg} / \mathrm{kg}$ on Day 28 (Fig. 5A). Initial recovery of plasma FXII was observed on Day 56 for the 3 $\mathrm{mg} / \mathrm{kg}$ dosage group, with FXII levels returning to baseline by $\sim 160$ d post-dose (Fig. 5B).

\section{FXII-dependent HK cleavage in human plasma}

Activation of the contact pathway leads to the cleavage of full-length high-molecular weight kininogen (HK) and the release of vasodilator bradykinin. HK cleavage can be evaluated by the detection of the disappearance of full-length HK upon contact pathway activation (Reddigari and Kaplan 1988; Baroso et al. 2016; Banerji et al. 2017). Evaluating the correlation between human plasma FXII levels and the extent of HK cleavage could potentially provide guidance on the level of FXII knockdown needed for efficacy. The steady-state HK cleavage levels are $~ 50 \%$ in HAE patient plasma versus $\sim 8.3 \%$ in plasma from healthy individuals (Banerji et al. 2017). To investigate the correlation between FXII levels and the extent of HK cleavage, we developed an ex vivo assay system using kaolin to induce contact pathway activation. To mimic the level of steadystate contact pathway activation in HAE patient plasma, normal human plasma was incubated with $0.2 \mu \mathrm{g} / \mathrm{mL}$ Kaolin for $10 \mathrm{~min}$ at $37^{\circ} \mathrm{C}$. This mild contact activation condition led to $\sim 50 \%$ HK cleavage in normal pooled human plasma (Fig. 6, 100\% FXII). By mixing the normal and congenital FXII-deficient ( $<1 \%$ FXII activity, nondetectable from ELISA, data not shown) human plasma, we generated a gradient concentration of FXII in the plasma mixture. Under the mild Kaolin contact activation condition that was described above, the percentage of HK cleavage was highly correlated with the levels of FXII. 15\% FXII in the plasma resulted in $~ 23 \%$ HK cleavage, and in FXII- deficient plasma, there was no detectable HK cleavage (Fig. 6). These data support the hypothesis that reducing plasma FXII levels can potentially suppress bradykinin generation. The same experiment was also performed for PK-deficient plasma and similar results were observed (data not shown). Consistent results were also observed in plasma samples from cynomolgus monkeys treated with ALN-F12 (Supplemental Fig. S5).

\section{DISCUSSION}

Type I and II HAE is characterized by a lack of functional $\mathrm{C} 1 \mathrm{INH}$ that leads to the uncontrolled activation of contact pathway and excessive bradykinin generation. The contact pathway is initiated by auto-activation of FXII in contact with negatively charged surfaces, such as protein aggregates or polyphosphates. Other than fresh frozen plasma or C1-INH concentrate (Cinryze, Berinert, Haegarda) to supply functional $\mathrm{C} 1 \mathrm{NH}$, the other approved treatments for HAE also target the bradykinin generation pathway, including plasma kallikrein inhibitor (Kalbitor and Takhzyro) and B2R antagonist (Firazyr). There is compelling genetic evidence supporting the correlation between plasma FXII levels and HAE clinical phenotypes. The F12-46C/T polymorphism is associated with decreased plasma FXII levels at $~ 50 \%$ (Kanaji et al. 1998), and F12-46C/T carriers exhibited a $\sim 7$-yr delay on disease onset decreased demand for long-term treatment

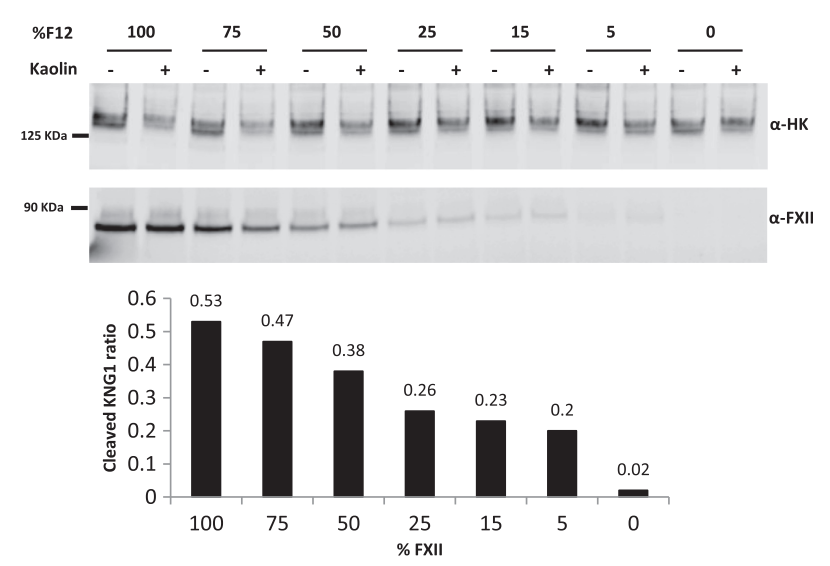

FIGURE 6. FXII dose-dependent HK cleavage in human plasma. Normal human pooled plasma was mixed with FXII-deficient $(<1 \%)$ human plasma at different ratios to generate different concentration of FXII in the mixture. The plasma mixtures were activated under mild conditions $\left(0.2 \mu \mathrm{g} / \mathrm{mL}\right.$ Kaolin, $\left.37^{\circ} \mathrm{C}, 10 \mathrm{~min}\right)$ and loaded into SDS-PAGE for western blot analysis. The anti-HK antibody detects the intact, full-length HK. The uncleaved full-length HK signal ratio of kaolin treatment to no kaolin treatment is represented in Table 1 below. 
TABLE 1. Designs and sequences of the GalNAc-siRNA conjugates

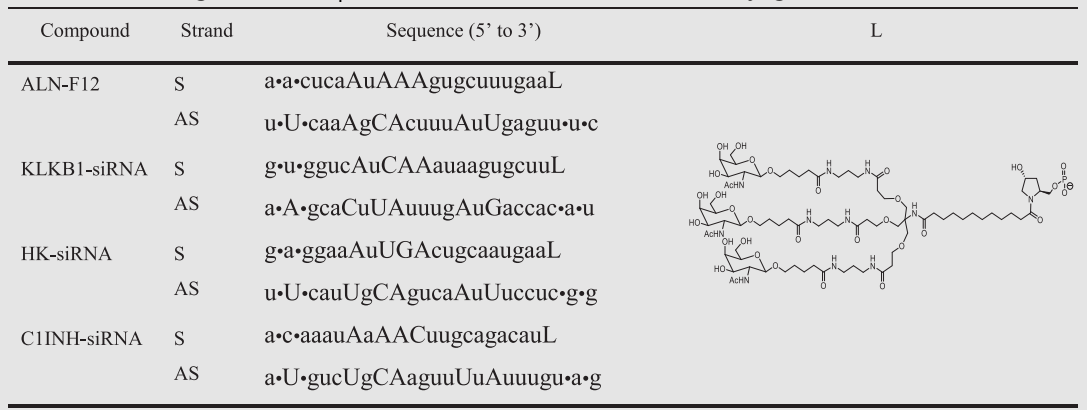

$\mathrm{S}$ and $\mathrm{AS}$ represent sense and antisense strands; upper case and lower case letters indicate $2^{\prime}$ deoxy-2'-fluoro $\left(2^{\prime}-\mathrm{F}\right)$, and $2^{\prime}$-O-methyl $\left(2^{\prime}-\mathrm{OMe}\right)$ ribosugar modifications, respectively; $(\bullet)$ indicates PS linkage. $L$ indicates the trivalent GalNAc ligand (structure above). the superior effect of FXII knockdown over PK or HK suppression in the mustard oil stimulation model in animals with reduced $\mathrm{C} 1 \mathrm{INH}$ levels. The superiority of FXII reduction over PK (or HK) in the ACE inhibitor model is a unique observation and this might be due to the experimental setting and mice genetic background.

Since many aspects of HAE attacks are not well understood, such as the triggers, the attack sites and the extent of FXII activation, it is difficult to predict how much suppression of plasma FXII would be sufficient to prevent HAE attacks. To explore the level of regardless of C1INH mutational status (Kanaji et al. 1998; Grunbacher et al. 2005; Bors et al. 2013; Speletas et al. 2015). Furthermore, among HAE patients with normal $\mathrm{C} 1 \mathrm{INH}$, also known as type III HAE, several distinct mutations in FXII have been identified. These FXII mutations, such as Thr309Lys, lead to the loss of glycosylation in FXII which results in the "gain-of-function" of FXIla and excess activation of the bradykinin generation pathway (Cichon et al. 2006; de Maat et al. 2016).

Using our GalNAc-conjugate siRNA delivery platform, we developed ALN-F12, a potent and stable GalNAcsiRNA duplex to efficiently target liver F12 mRNA and diminish plasma FXII levels. ALN-F12 has demonstrated robust potency and durability in both rodents and nonhuman primates. The durability of the pharmacodynamic effect of ALN-F12, and other GalNAc-siRNAs, is presumed to be due to the durability of the assembled RISC as well as the enhanced metabolic stability of the GalNAc-siRNA that allows for an extended duration of functional RISC loading (Foster et al. 2018). Off-target effects of ALNF12 were mitigated through both sequence-based bioinformatics and screening approaches as well as through the use of chemical modifications to abrogate innate immune responses. These approaches have been successfully applied in prior GalNAc-siRNA compounds evaluated in human clinical trials (Zimmermann et al. 2017).

To explore the effect of ALN-F12 on in vascular permeability, we implemented a modified ACE inhibitor stimulated vascular leakage model in mice (Han et al. 2002; Bhattacharjee et al. 2013). In this model we knocked down FXII, PK, or HK at different levels using corresponding GalNAc-siRNAs, and observed the mitigation effect on vascular leakage. PK and HK silencing was evaluated to determine whether other genes in the pathway may be alternative therapeutic targets. Interestingly, $\geq 50 \%$ F12 mRNA knockdown fully rescued the vascular leakage to peripheral tissues, while PK and HK mRNAs needed to be reduced to higher extents ( $>75 \%$ and $90 \%$, respectively) to achieve an equivalent effect. However, we did not observe
FXII reduction that may have an impact on HAE attacks, we developed an ex vivo human plasma assay to evaluate the correlation between FXII levels and bradykinin generation. In the anti-kallikrein antibody DX-2930 Phase 1b trial, HAE attacks were significantly decreased during Day 8 to Day 50 and $\sim 15 \%-25 \%$ of plasma HK was cleaved during this period compared to a steady-state activation level of $50 \%$ in HAE plasma (Banerji et al. 2017). In our ex vivo assay, FXII levels of $15 \%$ of normal led to $25 \%$ HK cleavage, which is comparable to the level of HK cleavage achieved in the DX-2930 trial. These data suggest that $>85 \%$ F12 knockdown may be sufficient to mitigate the frequency or severity of HAE attacks. This level of knockdown is achievable with subcutaneous administration of ALN-F12, based on both the efficacy of ALN-F12 in NHP, and the preliminary results from other Alnylam clinical programs utilizing GalNAc-siRNA conjugates (Fitzgerald et al. 2017a,b; Pasi et al. 2017; Zimmermann et al. 2017).

It is encouraging that human homozygous F12 deficiency (Hageman trait) has been described and has not been associated with increased bleeding tendency or other overt clinical phenotype. However, the safety of longterm suppression of FXII by ALN-F12 remains to be established in clinical trials. Furthermore, beyond a role in HAE, in recent years reports have suggested an important role of FXII in thrombosis and inflammation (Matafonov et al. 2014; Kenne et al. 2015), Alzheimer's disease (Zamolodchikov et al. 2016), and wound healing (Stavrou et al. 2018). Therefore, ALN-F12 mediated FXII lowering may potentially have broad clinical utility.

\section{MATERIALS AND METHODS}

\section{Care and use of laboratory animals}

Mice and rats were purchased from the Jackson Laboratory. All rodent studies were conducted at Alnylam Pharmaceuticals, an AALAS accredited animal facility, using protocols consistent with local, state and federal regulations and approved by the 
Institutional Animal Care and Use Committee (IACUC) at Alnylam Pharmaceuticals. Procedures using female cynomolgus monkeys were conducted by WIL Research/Charles River Laboratories, Ohio (2.5-5 yr of age; $2.3-5$ kg of bodyweight; Study No. WIL268503).

\section{Design and synthesis of ALN-F12 and other GalNAc-siRNAs targeting mouse PK, HK, and $\mathrm{C} 1 \mathrm{INH}$}

ALN-F12 is an investigational RNA interference (RNAi) therapeutic designed and synthesized by Alnylam Pharmaceuticals. The siRNA was designed to reduce liver production of Factor XII (FXII) by targeting a 23-nt region of F12 mRNA conserved among mouse, rat, cynomolgus monkey, and human. The drug substance of ALN-F12 is a chemically synthesized double-stranded oligonucleotide covalently linked to a ligand containing three $\mathrm{N}$-acetylgalactosamine (GalNAc) residues to enable delivery to hepatocytes via binding with asialoglycoprotein receptor (ASGPR) followed by clathrin-mediated endocytosis. The other GalNAcsiRNAs designed and synthesized by Alnylam Pharmaceuticals for this publication are as follows: PK-siRNA (NM_008455), HKsiRNA (NM_001102409), and C1INH-siRNA (NM_009776). Sequences and chemistry details of GalNAc-siRNA conjugates are listed in Table 1. Quality control parameters of the GalNAc-siRNA conjugates are summarized in Supplemental Table S2. Design of siRNA duplexes used an algorithm for chemical modification to optimize metabolic stability and intrinsic RNAi activity (Foster et al. 2018). For in vivo studies, all GalNAc-siRNAs were diluted in sterile $1 \times$ PBS and subcutaneously injected into the animals at $10 \mu \mathrm{L} / \mathrm{g}$ body weight for mice, $5 \mu \mathrm{L} / \mathrm{g}$ body weight for rats and $2 \mu \mathrm{L} / \mathrm{g}$ body weight for monkeys.

\section{Liver RT-OPCR and plasma FXII ELISA}

Mouse or rat liver tissues were harvested and snap frozen in liquid nitrogen to prevent mRNA degradation. Frozen liver tissue was ground, and tissue powder ( $20 \mathrm{mg}$ ) was lysed in $700 \mu \mathrm{L} \mathrm{RLT}$ lysis buffer provided from the RNeasy Mini Kit (QIAGEN). mRNA was extracted according to the manufacturer's instructions. The extracted RNA was eluted in $50 \mu \mathrm{L}$ of water. $10 \mu \mathrm{L}$ of eluted RNA $(\sim 2 \mu \mathrm{g})$ was used for cDNA synthesis utilizing a High Capacity cDNA synthesis kit (Thermo Fisher). Synthesis cDNA $(2 \mu \mathrm{L})$ was used for MultiPlex qPCR reactions. GAPDH was used as an internal control.

Plasma FXII total antigen levels were evaluated using ELISA kits from Molecular Innovations (mouse: MFXIIKT-TOT; rat: RFXIIKT-TOT; monkey: HFXIIKT-TOT) following the manufacturer's instructions. Briefly, K2EDTA-collected plasma samples were thawed on ice and diluted (mouse: 1:20,000; rat: 1:10,000; monkey: 1:2,000) with blocking buffer (3\% BSA, 100 mM Tris$\mathrm{HCl}, \mathrm{pH} 7.4,150 \mathrm{mM} \mathrm{NaCl}$ ). $100 \mu \mathrm{L}$ of diluted sample (or standard) was added to capture antibody-coated plate and incubated, followed by detection antibody and HRP-conjugated secondary antibody incubation, with washing steps included in between. The calculated plasma FXII concentrations for the test groups were then normalized to the average of the PBS-treated group or the corresponding individual animal's pre-dose average.

\section{ACE inhibitor-induced vascular permeability model}

Female C57BL/6J mice were subcutaneously (SC) administered a single dose of ALN-F12 at $0.1,0.3,1$, or $3 \mathrm{mg} / \mathrm{kg}(n=10$ per group). Seven days after ALN-F12 administration, animals were intravenously (IV) injected with $2.5 \mathrm{mg} / \mathrm{kg}$ ACE inhibitor captopril (Sigma-Aldrich), dissolved in $1 \times$ PBS at $10 \mu \mathrm{L} / \mathrm{g}$ body weight. The vascular leakage inert tracer, Evans blue dye $(30 \mathrm{mg} / \mathrm{kg}$ in $1 \times$ PBS), was IV injected in a tail vein 15 min after captopril injection. Animals were euthanized 15 min after Evans blue dye injection. To assess vascular permeability, $100 \mu \mathrm{L}$ of blood, collected via cardiac puncture, was mixed with $300 \mu \mathrm{L}$ ice-chilled acetone. After mixing thoroughly, the mixtures were centrifuged at $14,000 \mathrm{rpm}$ for $5 \mathrm{~min}$. Supernatant $(150 \mu \mathrm{L})$ was transferred to a 96-well flat-bottom plate for absorbance measurements at $620 \mathrm{~nm}$. A section of intestine $(\sim 15 \mathrm{~cm})$ was open longitudinally, rinsed twice in $0.9 \%$ sodium chloride, and transferred into balanced formamide (Sigma-Aldrich,) for Evans blue dye extraction. The tubes were incubated overnight at $55^{\circ} \mathrm{C}$, mixed, and centrifuged at 14,000 rpm for $10 \mathrm{~min}$. Supernatant $(150 \mu \mathrm{L})$ was then transferred to a 96-well flat-bottom plate for absorbance measurements at $620 \mathrm{~nm}$. Livers were collected for mRNA analysis.

\section{Mustard oil-induced vascular permeability model in C1INH knockdown mice}

The in vivo potency of the GalNAc-siRNA targeting mouse $\mathrm{C} 1 \mathrm{INH}$ (C1INH-siRNA) was confirmed (>95\% silencing of liver $\mathrm{C} 1-\mathrm{INH}$ mRNA, Supplemental Fig. S4B). Female CD-1 mice were given a single SC dose of a C1INH-siRNA at $10 \mathrm{mg} / \mathrm{kg}$ followed by a single dose of ALN-F12 (or PK-siRNA or HK-siRNA) at different doses within $10 \mathrm{~min}$ ( $n=10$ per group). Seven days after GalNAc-siRNAs administration, animals were IV injected in the tail vein with Evans blue dye (30 mg/kg in $1 \times$ PBS at $10 \mu \mathrm{L} / \mathrm{g}$ body weight). Immediately thereafter, 5\% mustard oil (Sigma-Aldrich, diluted with Mineral oil) was topically applied to the right ear of each animal followed by a second mustard oil application to the same ear 15 min later. The left ear remained untreated and served as the negative control. The animals were euthanized $30 \mathrm{~min}$ after the injection of Evans blue dye. To evaluate vascular permeability, each ear was collected, weighed, minced and $1 \mu \mathrm{L} / \mathrm{mg}$ tissue balanced formamide added for dye extraction as above. Livers were collected for mRNA analysis.

\section{Plasma HK cleavage assay}

Direct measurement of bradykinin generation is burdensome due to the instability and very short half-life $(<1 \mathrm{~min})$ of bradykinin (Murphey et al. 2000; Marketou and Vardas 2012). Instead, the levels of bradykinin generation can be quantified by plasma western blot using antibodies detecting the full-length, heavy chain, or light chain of HK. Normal pool and congenital FXII-deficient human plasma were purchased from George King Bio-Medical (Cat. No. 0010 and 1200). Cynomolgus plasma was obtained from animals dosed with ALN-F12 (Study No. WIL268503). In a 96-well PCR plate, $18 \mu \mathrm{L}$ Kaolin solution (Kaolin: Sigma-Aldrich, K7375; prepared in HBS buffer [20 mM HEPES, pH7.5, 140 mM $\mathrm{NaCl}$ ) was added into each well followed by the addition of $2 \mu \mathrm{L}$ plasma. The plate was put on a shaking incubator at $37^{\circ} \mathrm{C}$ 
for precisely $10 \mathrm{~min}$. Samples containing an equivalent of $0.5 \mu \mathrm{L}$ of plasma were loaded on denaturing NuPAGE SDS-PAGE gels (Invitrogen) for Western blot analysis using full-length HK and FXII antibodies from Abcam ( $\alpha$-HK: ab79650; $\alpha$-FXII: ab196670). A LiCoR Odymmey Infrared Imager (Li-COR Biosciences) was applied for quantification.

\section{SUPPLEMENTAL MATERIAL}

Supplemental material is available for this article.

Received September 20, 2018; accepted November 20, 2018.

\section{REFERENCES}

Banerji A, Busse P, Shennak M, Lumry W, Davis-Lorton M, Wedner HJ, Jacobs J, Baker J, Bernstein JA, Lockey R, et al. 2017. Inhibiting plasma kallikrein for hereditary angioedema prophylaxis. N Engl J Med 376: 717-728. doi:10.1056/NEJMoa1605767

Baroso R, Sellier P, Defendi F, Charignon D, Ghannam A, Habib M, Drouet C, Favier B. 2016. Kininogen cleavage assay: diagnostic assistance for kinin-mediated angioedema conditions. PLoS One 11: e0163958. doi:10.1371/journal.pone.0163958

Bhattacharjee G, Revenko AS, Crosby JR, May C, Gao D, Zhao C, Monia BP, MacLeod AR. 2013. Inhibition of vascular permeability by antisense-mediated inhibition of plasma kallikrein and coagulation factor 12. Nucleic Acid Ther 23: 175-187. doi:10.1089/ nat.2013.0417

Björkqvist J, Nickel KF, Stavrou E, Renné T. 2014. In vivo activation and functions of the protease factor XII. Thromb Haemost 112: 868-875. doi:10.1160/th14-04-0311

Björkqvist J, de Maat S, Lewandrowski U, Di Gennaro A, Oschatz C, Schönig K, Nöthen MM, Drouet C, Braley H, Nolte MW, et al. 2015. Defective glycosylation of coagulation factor XII underlies hereditary angioedema type III. J Clin Invest 125: 3132-3146. doi:10.1172/JCl77139

Bork K. 2013. Hereditary angioedema with normal C1 inhibitor. Immunol Allergy Clin North Am 33: 457-470. doi:10.1016/j. iac.2013.07.002

Bork K, Davis-Lorton M. 2013. Overview of hereditary angioedema caused by C1-inhibitor deficiency: assessment and clinical management. Eur Ann Allergy Clin Immunol 45: 7-16.

Bork K, Wulff K, Hardt J, Witzke G, Staubach P. 2009. Hereditary angioedema caused by missense mutations in the factor XII gene: clinical features, trigger factors, and therapy. J Allergy Clin Immunol 124: 129-134. doi:10.1016/j.jaci.2009.03.038

Bors A, Csuka D, Varga L, Farkas H, Tordai A, Füst G, Szilagyi A. 2013. Less severe clinical manifestations in patients with hereditary angioedema with missense C1INH gene mutations. J Allergy Clin Immunol 131: 1708-1711. doi:10.1016/j.jaci.2012.11.015

Carugati A, Pappalardo E, Zingale LC, Cicardi M. 2001. C1-inhibitor deficiency and angioedema. Mol Immunol 38: 161-173. doi:10.1016/S0161-5890(01)00040-2

Cichon S, Martin L, Hennies HC, Müller F, Van Driessche K, Karpushova A, Stevens W, Colombo R, Renné T, Drouet C, et al. 2006. Increased activity of coagulation factor XII (Hageman factor) causes hereditary angioedema type III. Am J Hum Genet 79: 1098-1104. doi:10.1086/509899

de Maat S, Björkqvist J, Suffritti C, Wiesenekker CP, Nagtegaal W, Koekman A, van Dooremalen S, Pasterkamp G, de Groot PG, Cicardi $M$, et al. 2016. Plasmin is a natural trigger for bradykinin production in patients with hereditary angioedema with factor
XII mutations. J Allergy Clin Immunol 138: 1414-1423.e9. doi:10.1016/j.jaci.2016.02.021

Fitzgerald K, Kallend D, Simon A. 2017a. A highly durable RNAi therapeutic inhibitor of PCSK9. N Engl J Med 376: e38. doi:10.1056/ NEJMc1703361

Fitzgerald K, White S, Borodovsky A, Bettencourt BR, Strahs A, Clausen V, Wijngaard P, Horton JD, Taubel J, Brooks A, et al. 2017b. A highly durable RNAi therapeutic inhibitor of PCSK9. N Engl J Med 376: 41-51. doi:10.1056/NEJMoa1609243

Foster DJ, Brown CR, Shaikh S, Trapp C, Schlegel MK, Qian K, Sehgal A, Rajeev KG, Jadhav V, Manoharan M, et al. 2018. Advanced siRNA designs further improve in vivo performance of GalNAc-siRNA conjugates. Mol Ther 26: 708-717. doi:10.1016/ j.ymthe.2017.12.021

Grunbacher G, Marx-Neuhold E, Pilger E, Koppel H, Renner W. 2005. The functional $-4 \mathrm{C}>\mathrm{T}$ polymorphism of the coagulation factor XII gene is not associated with deep venous thrombosis. J Thromb Haemost 3: 2815-2817. doi:10.1111/j.1538-7836.2005.01651.x

Han ED, MacFarlane RC, Mulligan AN, Scafidi J, Davis AE III. 2002. Increased vascular permeability in $\mathrm{C} 1$ inhibitor-deficient mice mediated by the bradykinin type 2 receptor. J Clin Invest 109: 10571063. doi: $10.1172 / \mathrm{JCl} 200214211$

Kanaji T, Okamura T, Osaki K, Kuroiwa M, Shimoda K, Hamasaki N, Niho Y. 1998. A common genetic polymorphism (46 C to T substitution) in the $5^{\prime}$-untranslated region of the coagulation factor XII gene is associated with low translation efficiency and decrease in plasma factor XII level. Blood 91: 2010-2014.

Kenne E, Nickel KF, Long AT, Fuchs TA, Stavrou EX, Stahl FR, Renné T. 2015. Factor XII: a novel target for safe prevention of thrombosis and inflammation. J Intern Med 278: 571-585. doi:10.1111/joim. 12430

Marketou ME, Vardas PE. 2012. Bradykinin in the treatment of arterial hypertension: friend or foe? Hellenic J Cardiol 53: 91-94.

Matafonov A, Leung PY, Gailani AE, Grach SL, Puy C, Cheng Q, Sun MF, McCarty OJ, Tucker El, Kataoka H, et al. 2014. Factor $\mathrm{XII}$ inhibition reduces thrombus formation in a primate thrombosis model. Blood 123: 1739-1746. doi:10.1182/blood-2013-04499111

Matsuda S, Keiser K, Nair JK, Charisse K, Manoharan RM, Kretschmer P, Peng CG, V Kel'in A, Kandasamy P, Willoughby JL, et al. 2015. siRNA conjugates carrying sequentially assembled trivalent $\mathrm{N}$-acetylgalactosamine linked through nucleosides elicit robust gene silencing in vivo in hepatocytes. ACS Chem Biol 10: 1181-1187. doi:10.1021/cb501028c

Migdalof BH, Antonaccio MJ, McKinstry DN, Singhvi SM, Lan SJ, Egli P, Kripalani KJ. 1984. Captopril: pharmacology, metabolism and disposition. Drug Metab Rev 15: 841-869. doi:10.3109/ 03602538409041080

Müller F, Mutch NJ, Schenk WA, Smith SA, Esterl L, Spronk HM, Schmidbauer S, Gahl WA, Morrissey JH, Renné T. 2009. Platelet polyphosphates are proinflammatory and procoagulant mediators in vivo. Cell 139: 1143-1156. doi:10.1016/j.cell.2009.11.001

Murphey LJ, Hachey DL, Oates JA, Morrow JD, Brown NJ. 2000. Metabolism of bradykinin in vivo in humans: identification of BK1-5 as a stable plasma peptide metabolite. J Pharmacol Exp Ther 294: 263-269.

Nair JK, Willoughby JL, Chan A, Charisse K, Alam MR, Wang Q, Hoekstra M, Kandasamy P, Kel'in AV, Milstein S, et al. 2014. Multivalent $\mathrm{N}$-acetylgalactosamine-conjugated siRNA localizes in hepatocytes and elicits robust RNAi-mediated gene silencing. J Am Chem Soc 136: 16958-16961. doi:10.1021/ja505986a

Nussberger J, Cugno M, Cicardi M. 2002. Bradykinin-mediated angioedema. N Engl J Med 347: 621-622. doi:10.1056/NEJM 200208223470820 
Pappalardo E, Zingale LC, Terlizzi A, Zanichelli A, Folcioni A, Cicardi M. 2002. Mechanisms of C1-inhibitor deficiency. Immunobiology 205: 542-551. doi:10.1078/0171-2985-00153

Pasi KJ, Rangarajan S, Georgiev P, Mant T, Creagh MD, Lissitchkov T, Bevan D, Austin S, Hay CR, Hegemann I, et al. 2017. Targeting of antithrombin in hemophilia A or B with RNAi therapy. N Engl J Med 377: 819-828. doi:10.1056/NEJMoa1616569

Reddigari S, Kaplan AP. 1988. Cleavage of human high-molecular weight kininogen by purified kallikreins and upon contact activation of plasma. Blood 71: 1334-1340.

Schmaier AH. 2000. Plasma kallikrein/kinin system: a revised hypothesis for its activation and its physiologic contributions. Curr Opin Hematol 7: 261-265. doi:10.1097/00062752-20000900000001

Speletas M, Szilágyi A, Csuka D, Koutsostathis N, Psarros F, Moldovan D, Magerl M, Kompoti M, Varga L, Maurer M, et al. 2015. F12-46C/T polymorphism as modifier of the clinical phenotype of hereditary angioedema. Allergy 70: 1661-1664. doi:10.1111/all.12714

Stavrou EX, Fang C, Bane KL, Long AT, Naudin C, Kucukal E, Gandhi A, Brett-Morris A, Mumaw MM, Izadmehr S, et al. 2018. Factor XII and uPAR upregulate neutrophil functions to influence wound healing. J Clin Invest 128: 944-959. doi:10.1172/ $\mathrm{JC} 192880$
Walford HH, Zuraw BL. 2014. Current update on cellular and molecular mechanisms of hereditary angioedema. Ann Allergy Asthma Immunol 112: 413-418. doi:10.1016/j.anai.2013.12.023

Zamolodchikov D, Renné T, Strickland S. 2016. The Alzheimer's disease peptide $\beta$-amyloid promotes thrombin generation through activation of coagulation factor XII. J Thromb Haemost 14: 9951007. doi:10.1111/jth.13209

Zhang JC, Claffey K, Sakthivel R, Darzynkiewicz Z, Shaw DE, Leal J, Wang YC, Lu FM, McCrae KR. 2000. Two-chain high molecular weight kininogen induces endothelial cell apoptosis and inhibits angiogenesis: partial activity within domain 5. FASEB $J$ 14: 2589-2600. doi:10.1096/fj.99-1025com

Zimmermann TS, Karsten V, Chan A, Chiesa J, Boyce M, Bettencourt BR, Hutabarat R, Nochur S, Vaishnaw A, Gollob J. 2017. Clinical proof of concept for a novel hepatocyte-targeting GalNAc-siRNA conjugate. Mol Ther 25: 71-78. doi:10.1016/j.ymthe. 2016.10.019

Zuraw BL. 2010. Summary and conclusions: new perspectives in hereditary angioedema: molecular mechanisms and therapeutic choices: a CME symposium presented at the World Allergy Congress, Buenos Aires, Argentina (December 2009). World Allergy Organ J 3: S39-S40. doi:10.1186/1939-4551-3-S3-S39

Zuraw BL, Christiansen SC. 2011. Pathophysiology of hereditary angioedema. Am J Rhinol Allergy 25: 373-378. doi:10.2500/ajra. 2011.25.3661 

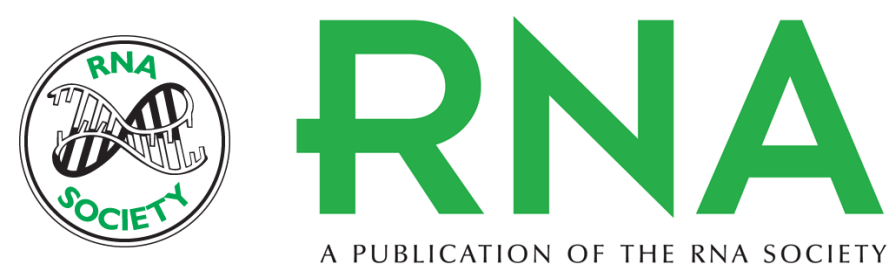

A PUBLICATION OF THE RNA SOCIETY

\section{An investigational RNAi therapeutic targeting Factor XII (ALN-F12) for the treatment of hereditary angioedema}

Jingxuan Liu, June Qin, Anna Borodovsky, et al.

RNA 2019 25: 255-263 originally published online November 21, 2018

Access the most recent version at doi:10.1261/rna.068916.118

\section{Supplemental http://rnajournal.cshlp.org/content/suppl/2018/11/21/rna.068916.118.DC1 Material}

References This article cites 39 articles, 4 of which can be accessed free at: http://rnajournal.cshlp.org/content/25/2/255.full.html\#ref-list-1

Creative This article is distributed exclusively by the RNA Society for the first 12 months after the Commons License full-issue publication date (see http://rnajournal.cshlp.org/site/misc/terms.xhtml). After 12 months, it is available under a Creative Commons License (Attribution-NonCommercial 4.0 International), as described at http://creativecommons.org/licenses/by-nc/4.0/.

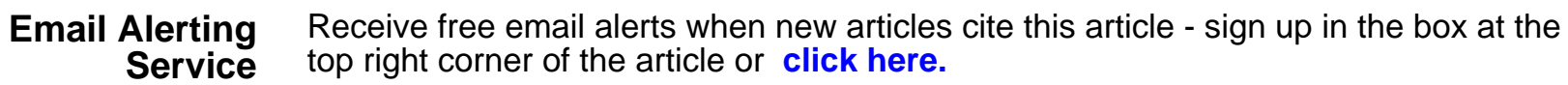

